STRUCTURAL BIOLOGY COMMUNICATIONS

ISSN 2053-230X

\section{Conformational flexibility in the zinc solute-binding protein ZnuA}

\author{
Elsie Laban Yekwa, Fred Allen Serrano and Erik Yukl*
}

Department of Chemistry and Biochemistry, New Mexico State University, 1175 North Horseshoe Drive, Las Cruces, NM 88003, USA. *Correspondence e-mail: etyukl@nmsu.edu

Received 3 December 2021

Accepted 10 February 2022

Edited by N. Sträter, University of Leipzig, Germany

Keywords: zinc; ZnuA; Citrobacter koseri; solute-binding proteins; ATP-binding cassette transporters.

PDB reference: ZnuA from Citrobacter koseri, 7rcj

Supporting information: this article has supporting information at journals.iucr.org/f
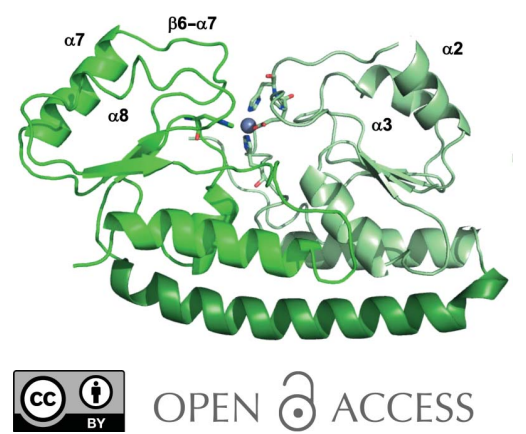

Published under a CC BY 4.0 licence
Zinc is an essential metal for all kingdoms of life, making its transport across the cell membrane a critical function. In bacteria, high-affinity zinc import is accomplished by ATP-binding cassette (ABC) transporters, which rely on extracellular solute-binding proteins (SBPs) of cluster A-I to acquire the metal and deliver it to the membrane permease. These systems are important for survival and virulence, making them attractive targets for the development of novel antibiotics. Citrobacter koseri is an emerging pathogen with extensive antibiotic resistance. High-affinity zinc binding to the C. koseri cluster A-I SBP ZnuA has been characterized and the structure of the zinc-bound (holo) form has been determined by X-ray crystallography. Remarkably, despite 95\% sequence identity to the ZnuA homologue from Salmonella enterica, C. koseri ZnuA exhibits a different zinc-coordination environment and a closed rather than an open conformation. Comparison with structures of another close ZnuA homologue from Escherichia coli suggests a surprisingly flexible conformational landscape that may be important for efficient zinc binding and/or delivery to the membrane permease.

\section{Introduction}

The ATP-binding cassette $(\mathrm{ABC})$ transporters are a superfamily involved in the translocation of numerous substrates across the cell membrane using ATP hydrolysis (Higgins, 1992). In addition to the membrane permease and cytoplasmic ATPase, bacterial importers of this type also require a solutebinding protein (SBP) in the periplasm or outer membrane (Davidson et al., 2008; van der Heide \& Poolman, 2002) to specifically and efficiently bind and deliver the substrate (Khare et al., 2009). Upon binding to the cognate substrate, the SBP is generally thought to convert from an open to a closed conformation, which is important for recognition by the membrane permease and subsequent transport (Davidson et al., 2008; Quiocho \& Ledvina, 1996). However, the degree of conformational change varies dramatically among different SBPs and substrates, with some exhibiting very little change (Berntsson et al., 2010). Further, recent solution work has shown that dynamic interconversion between an ensemble of open and closed states is important for the specific transport of cognate substrates (de Boer et al., 2019). Thus, a simple twostate mechanism for permease recognition and cognate substrate transport is likely to be an oversimplification for many ABC transporters.

SBPs have been classified into seven clusters (A-G) based on structure, with further subdivisions based on substrate specificity (Berntsson et al., 2010, Scheepers et al., 2016). By this classification, SBPs that bind zinc, manganese or iron 
belong to cluster A-I, which is defined by two related $\alpha / \beta$ domains linked by a long $\alpha$-helix. Within this cluster, sequence similarity identifies several groups that differ on the basis of substrate specificity, coordination environment and the presence of a long flexible loop region near the substratebinding site (Loisel et al., 2008). The ZnuA proteins of Gramnegative species primarily belong to group II, which is defined by zinc specificity, the presence of a long flexible loop rich in His and Asp/Glu residues, and metal coordination by three His residues and one Glu residue or water in a tetrahedral geometry. Deletion mutants show diminished infection potential and virulence in infection models for several human pathogens including Salmonella enterica (Ammendola et al., 2007), Vibrio cholerae (Sheng et al., 2015), Brucella abortus (Yang et al., 2006) and Acinetobacter baumanii (Hesse et al., 2019), highlighting the potential of these proteins as antibiotic drug targets. Indeed, a lead compound was identified that bound to ZnuA from S. enterica and inhibited the growth of this organism (Ilari et al., 2016). However, to our knowledge this was never developed for clinical use. While the early indications are promising, the effective exploitation of zinc solute-binding proteins will likely require detailed structural and mechanistic data.

Several crystal structures of cluster A-I SBPs in group II have been solved, including some in both holo and apo states. ZnuA from Escherichia coli (Ec ZnuA) shows an open conformation in the apo state, and zinc binding elicits conversion to the closed state primarily through motions of the C-terminal domain (CTD), with the zinc coordinated by Glu59, His60, His143 and His207 (Yatsunyk et al., 2008; Li \& Jogl, 2007). The holo structure of Synechocystis ZnuA is also closed, but the coordinating Glu residue is not conserved and a water molecule occupies the fourth coordination site (Banerjee et al., 2003). Finally, holo S. enterica ZnuA (Se ZnuA) exhibits an open conformation and His60 is replaced by a residue from the flexible loop, His140 (Ilari et al., 2011). Apo structures of deletion mutants of the flexible loops for the latter two proteins are in closed conformations (Wei et al., 2007). Thus, there appears to be significant flexibility in coordination environment and conformation among the ZnuA proteins, which may reflect dynamics that are important for substrate specificity and permease recognition.

Here, we characterize zinc binding to the cluster A-I SBP ZnuA from the human pathogen Citrobacter koseri ( $\mathrm{Ck}$ ZnuA) and present the crystal structure of the holo form. C. koseri is a Gram-negative organism that is associated with an increasing number of primarily nosocomial infections. Further, the incidence of multidrug resistance in Citrobacter species is widespread and rising, including resistance to the last-resort antibiotic class of carbapenems (Yao et al., 2021). This severely limits treatment options and highlights the importance of identifying new antibiotic drug targets in this organism. $C k$ ZnuA is highly homologous to ZnuA proteins from S. enterica and E. coli, with $95 \%$ and $85 \%$ sequence identity, respectively, excluding the periplasmic targeting sequences. Despite this, these proteins exhibit a remarkable diversity of structures and metal-coordination environments between them, allowing us to present an ensemble of structures in both apo and holo forms. The results indicate a surprisingly plastic zinc-binding environment for the ZnuA family.

\section{Materials and methods}

\subsection{Macromolecule production}

The intact gene encoding wild-type (WT) $C k$ ZnuA (UniProt ID A8AFI6) was amplified by PCR from C. koseri strain ATCC BAA-895 genomic DNA and cloned into pCDFDuet (Novagen) at NdeI and Acc65I/KpnI restriction sites. The ZnuA loop deletion lacking residues 124-143 ( $\triangle$ Loop ZnuA) was generated using the Q5 Site-Directed Mutagenesis Kit (New England BioLabs). Both plasmids were confirmed by sequencing. Both WT and $\triangle$ Loop ZnuA were expressed in E. coli BL21(DE3) cells grown in LB medium containing $50 \mu \mathrm{g} \mathrm{ml}^{-1}$ streptomycin at $37^{\circ} \mathrm{C}$ with shaking at $250 \mathrm{rev} \mathrm{min}^{-1}$ to an $\mathrm{OD}_{600}$ of $0.8-1.0$. Overexpression was induced by isopropyl $\beta$-D-1-thiogalactopyranoside at a concentration of $1.0 \mathrm{~m} M$ and the temperature was decreased to $20^{\circ} \mathrm{C}$. After overnight growth with shaking, the cells were harvested by centrifugation at $4000 \mathrm{~g}$ for $30 \mathrm{~min}$ at $4^{\circ} \mathrm{C}$.

The periplasmic fraction was prepared using an osmotic shock protocol adapted from Wang et al. (2003). Polyethylamineimine (PEI) was added to $0.5 \%(v / v)$ and centrifuged at $25000 \mathrm{~g}$ at $4^{\circ} \mathrm{C}$ for $20 \mathrm{~min}$. Ammonium sulfate was added to the supernatant to $70 \%$ saturation and centrifuged as above for $30 \mathrm{~min}$. The ammonium sulfate pellet was then drained and resolubilized in $20 \mathrm{mM}$ Tris $\mathrm{pH} 8.0$ and initially purified at this $\mathrm{pH}$ by anion-exchange chromatography (IEC) using a HiTrap Q HP column (Cytiva) with an increasing gradient of $\mathrm{NaCl}$. $\triangle$ Loop ZnuA was dialyzed against $20 \mathrm{mM}$ Tris $\mathrm{pH} 9.0$ and IEC was performed at this $\mathrm{pH}$. Fractions containing $C k$ ZnuA were combined, concentrated to $<1 \mathrm{ml}$ and further purified using a HiPrep Sephacryl S-200 HR sizeexclusion chromatography (SEC) column (Cytiva) equilibrated with $50 \mathrm{~m} M$ Tris $\mathrm{pH} 8.0,150 \mathrm{~m} M \mathrm{NaCl}$. After final purification by SEC, all proteins were highly pure as judged by SDS-PAGE. WT $C k$ ZnuA and $\triangle$ Loop $C k$ ZnuA concentrations were determined using extinction coefficients at $280 \mathrm{~nm}$ of 31162 and $28816 M^{-1} \mathrm{~cm}^{-1}$, respectively, calculated as described previously (Edelhoch, 1967). Macromolecule-production information is summarized in Table 1.

2.1.1. Generation of apoproteins and metal quantitation. Apoproteins were generated by dialysis at $4^{\circ} \mathrm{C}$ and complete zinc removal was confirmed by inductively coupled plasmaoptical emission spectrometry (ICP-OES) as described previously (Neupane et al., 2019). Buffer blanks were generated identically to protein samples using an equal volume of buffer relative to protein solution. Metal content was quantified using a Perkin-Elmer 2100 DV ICP-OES calibrated with a multi-element standard (Alpha Aesar) at a wavelength of $213.857 \mathrm{~nm}$ for zinc.

2.1.2. Mag-Fura-2 competition assay. Zinc-binding affinities were measured using a Mag-Fura-2 (MF-2) competition 
Table 1

Macromolecule-production information.

\begin{tabular}{ll}
\hline Source organism & Citrobacter koseri \\
DNA source & Citrobacter koseri genomic DNA \\
Forward primer & GCTCTCATATGATTAGTCGCATTATGTTAC \\
& ATAA \\
Reverse primer & ACTATGGTACCTTAATCTCCTTTCAGGCA \\
Cloning vector & pCDFDuet \\
Expression vector & pCDFDuet \\
Expression host & Escherichia coli BL21(DE3) \\
Complete amino-acid sequence & AVVASLKPLGFIASAIADGVTDTQVLLPDG \\
\multicolumn{1}{c}{ of the construct produced } & ASEHDYSLRPSDVRLQGADLVVIGPE \\
& MEAFMEKSVKNIPDGKQVTIAQLADVKP \\
& LLMKGADYNMHLWLSPEIARASAVAIHE \\
& KLVELMPQSRAKLDANLKDFEAQLAATD \\
& KQVGNELAPLKGKGYFVHDAYGYEKH \\
& YGLTPLGHFTVNPEIQPGAQRLHEIRTQ \\
& LVEQKATCVFAEPQFRPAVVEAVARGTS \\
& VRMGTLDPLGTNIKLGKTSYSAFLNQLA \\
& NQYASCLKG \\
\hline
\end{tabular}

assay derived from Golynskiy et al. (2006) using concentrations and instrument parameters as described previously (Neupane et al., 2019; Handali, Neupane et al., 2015; Handali, Roychowdhury et al., 2015). Experiments were performed in triplicate and the data were fitted using DYNAFIT (Kuzmič, 1996, 2006, 2009) and a script modified from that described previously (Handali, Neupane et al. 2015) to account for three zinc-binding sites in the protein. DYNAFIT uses simultaneous nonlinear algebraic equations to fit equilibrium data rather than a single binding isotherm expression. For $\Delta$ Loop $C k$ ZnuA, the calculated $K_{\mathrm{d}}$ values for sites 2 and 3 were greater than 100-fold that of MF-2, indicating that multiple metal binding could not be detected using this assay, and an equivalent fit was acquired using a single binding site. The affinity of MF-2 for zinc in our buffer system was determined prior to each series of experiments and the $K_{\mathrm{d}}$ varied from 59 to $110 \mathrm{n} M$.

\subsection{Crystallization}

Initial crystallization hits for WT $C k$ ZnuA were identified using the Hauptman-Woodward Institute standard screen (Luft et al., 2004). These were optimized in-house and diffraction-quality crystals were grown by vapor diffusion using a 1:1 ratio of $18 \mathrm{mg} \mathrm{ml}^{-1} C k$ ZnuA reconstituted with one equivalent of zinc and precipitant solution consisting of $0.1 M$ sodium citrate $\mathrm{pH}$ 6.0, $0.05 \mathrm{M}$ ammonium acetate, $1 \mathrm{~m} M$ hexatungstotellurate (TEW; Mauracher et al., 2014) incubated at $298 \mathrm{~K}$. TEW was synthesized in-house as described previously (Mauracher et al., 2014). The crystals were cryoprotected in reservoir solution containing 10\% PEG 400 and were cryocooled in liquid nitrogen. Crystallization information is summarized in Table 2.

\subsection{Data collection and processing}

Diffraction data were collected at $100 \mathrm{~K}$ on beamline 5.0.1 at the Advanced Light Source at Berkeley National Laboratory, indexed and integrated with $X D S$ version 0.92 (Kabsch, 2010a,b) and scaled using AIMLESS (Winn et al., 2011). Datacollection and processing statistics are summarized in Table 3.
Table 2

Crystallization.

\begin{tabular}{ll}
\hline Method & Vapor diffusion \\
Plate & VDX \\
Temperature (K) & 298 \\
Protein concentration $\left(\mathrm{mg} \mathrm{ml}^{-1}\right)$ & 18 \\
Protein buffer & $50 \mathrm{~m} M$ Tris $\mathrm{pH} 8.0,150 \mathrm{~m} M \mathrm{NaCl}$ \\
Reservoir composition & $0.1 M$ sodium citrate $\mathrm{pH} 6.0$, \\
& $0.05 M$ ammonium acetate, \\
& $1 \mathrm{~m} M$ hexatungstotellurate (TEW) \\
Drop volume and ratio & $2 \mu \mathrm{l}, 1: 1$ ratio \\
Reservoir volume $(\mu \mathrm{l})$ & 500 \\
\hline
\end{tabular}

Table 3

Data collection and processing.

Values in parentheses are for the outer shell.

\begin{tabular}{ll}
\hline Diffraction source & Beamline 5.0.1, ALS \\
Wavelength $(\AA)$ & 1.000 \\
Temperature $(\mathrm{K})$ & 100 \\
Detector & PILATUS3 6M 25 Hz \\
Crystal-to-detector distance $(\mathrm{mm})$ & 500 \\
Rotation range per image $\left({ }^{\circ}\right)$ & 1.0 \\
Total rotation range $\left({ }^{\circ}\right)$ & 200 \\
Exposure time per image $(\mathrm{s})$ & 1.0 \\
Space group & $P 2$ \\
$a, b, c(\AA)$ & $122.25,81.67,126.56$ \\
$\alpha, \beta, \gamma\left({ }^{\circ}\right)$ & $90,112.99,90$ \\
Mosaicity $\left({ }^{\circ}\right)$ & 0.31 \\
Resolution range $(\AA)$ & $47.5-3.15(3.28-3.15)$ \\
Total No. of reflections & $148165(16672)$ \\
No. of unique reflections & $39993(4489)$ \\
Completeness $(\%)$ & $99.8(99.8)$ \\
Multiplicity & $3.7(3.7)$ \\
$\langle I / \sigma(I)\rangle$ & $5.9(0.8) \dagger$ \\
$R_{\text {r.i.m. }} \dagger$ & $0.17(1.30)$ \\
CC & $0.99(0.34)$ \\
Overall $B$ factor from Wilson plot $\left(\AA^{2}\right)$ & 89.3
\end{tabular}

$\dagger$ A cutoff of $\mathrm{CC}_{1 / 2}>0.3$ was used as data below the $I / \sigma(I)$ cutoff were still valuable and improved the resolution, as discussed in the text.

\subsection{Structure solution and refinement}

The model of zinc-bound Se ZnuA (PDB entry 2xqv; Ilari et $a l ., 2011)$ lacking zinc and waters was used as the search model for molecular replacement using Phaser-MR (McCoy et al., 2007). Manual model building was performed in Coot (Emslet et al., 2010) and further rounds of refinement were performed in REFMAC (Murshudov et al., 2011). Atomic coordinates of zinc-bound $C k$ ZnuA have been deposited in the PDB as entry 7rcj. Figures were prepared using PyMOL (http:// www.pymol.org), which was also used for pairwise structural alignments of $C k$ ZnuA with other solute-binding proteins. Refinement statistics are summarized in Table 4.

\section{Results and discussion}

\subsection{Zinc binding to C. koseri ZnuA}

Since $C k$ ZnuA had not previously been characterized, the affinity and stoichiometry of zinc binding were determined using a competitive fluorescence assay with Mag-Fura-2 (MF2; Golynskiy et al., 2006; Fig. 1). We also investigated binding to a deletion mutant lacking a flexible, His-rich loop (residues 124143, $\Delta$ Loop $C k$ ZnuA) common to most zinc-specific SBPs. 
Table 4

Structure refinement.

Values in parentheses are for the outer shell.

\begin{tabular}{ll}
\hline Resolution range $(\AA)$ & $47.47-3.15(3.232-3.150)$ \\
Completeness (\%) & 99.7 \\
No. of reflections, working set & $37978(2771)$ \\
No. of reflections, test set & $2006(144)$ \\
Final $R_{\text {cryst }}$ & $0.217(0.365)$ \\
Final $R_{\text {free }}$ & $0.254(0.428)$ \\
No. of non-H atoms & \\
$\quad$ Protein & 12237 \\
$\quad$ Ion & 6 \\
$\quad$ Total & 12243 \\
R.m.s. deviations & \\
$\quad$ Bond lengths $(\AA)$ & 0.012 \\
$\quad$ Angles $\left({ }^{\circ}\right)$ & 1.643 \\
Average $B$ factors $\left(\AA^{2}\right)$ & \\
$\quad$ Protein & 106.2 \\
$\quad$ Ion & 89.4 \\
Ramachandran plot & \\
$\quad$ Favored regions $(\%)$ & 91.2 \\
$\quad$ Additionally allowed $(\%)$ & 5.7 \\
$\quad$ Outliers $(\%)$ & 3.0
\end{tabular}

The results show that $C k \mathrm{ZnuA}$ binds at least three zinc ions with high affinity. The observation that $\triangle$ Loop $C k$ ZnuA binds only a single zinc ion indicates that the formation of two additional zinc-binding sites requires the presence of the Hisrich loop. The large error on the lowest-affinity binding event is a consequence of weak binding affinity relative to the MF2 probe. Nevertheless, this assay confirms high-affinity zinc binding consistent with that observed for other cluster A-I SBPs.

\subsection{Crystal structure of C. koseri ZnuA}

Initial crystallization conditions yielded small, disordered crystals with poor diffraction. A screen of additives identified hexatungstotellurate (TEW; Mauracher et al., 2014) to contribute to larger, better-ordered crystals, as has previously been reviewed (Bijelic \& Rompel, 2017, 2018). These crystals belonged to space group $P 2$, with six copies of $C k \mathrm{ZnuA}$ in the asymmetric unit. Three copies of TEW are also evident at special positions, demonstrating how they are able to generate crystal contacts and stabilize the crystal lattice (Fig. 2a). With a formal charge of minus six, TEW often interacts electrostatically with positively charged residues and through hydrogen bonding in protein structures (Mac Sweeney et al., 2018; Vandebroek et al., 2020; Bijelic et al., 2015; Sobala et al., 2020). Each TEW in the $C k$ ZnuA structure is in close proximity to Lys91 of four symmetry-related protein chains (Fig. 2b). However, poor electron density around the side chains precludes the confident assignment of these residues as coordinating ligands.

Even with the stabilizing interactions provided by TEW, the resolution of the data was relatively poor, prompting us to use the more generous resolution cutoff based on $\mathrm{CC}_{1 / 2}$ (Karplus \& Diederichs, 2012, 2015; Table 3). The resulting electrondensity map was of good quality throughout, and the $R$ factors for the refined structures are relatively low, illustrating the utility of this approach. The average $B$ factors are unusually high, which is likely to be a consequence of the low resolution of the data and the relatively high solvent content $(\sim 64 \%)$ of these crystals. Finally, there are a relatively large number of Ramanchandran outliers, which are not typically observed in other ZnuA structures. Several of these are localized to helix $\alpha 8$ and are flagged as outliers in each of the six protein copies (for example residues 266, 271 and 272). While this suggests that these may actually exist as outliers, the low resolution of the data preclude us from ascribing any functional significance to this observation.

The overall structure of $C k$ ZnuA is similar to those of other cluster A-I SBPs solved to date and consists of an $\mathrm{N}$-terminal domain (NTD) and a C-terminal domain (CTD) connected by a long $\alpha$-helix (Fig. 2c). It is in the closed conformation with the $\beta 6-\alpha 7$ loop closed over the zinc site. All residues could be modeled into the electron density with the exception of the His-rich loop, which is typical of cluster A-I SBP structures, where this region is disordered. In all six

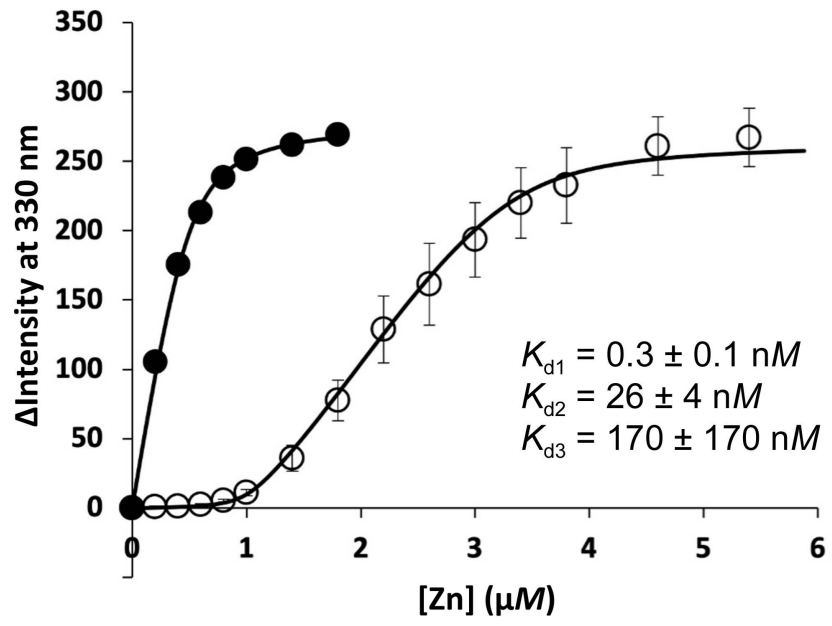

(a)

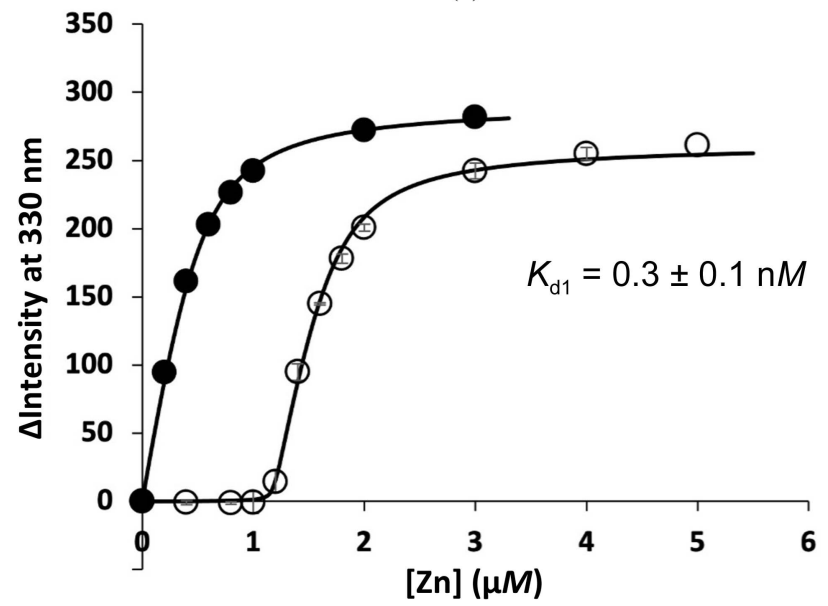

(b)

Figure 1

Zinc binding by $C$. koseri ZnuA determined by MF2 competition assay. The intensity change at $330 \mathrm{~nm}$ with increasing zinc in the absence (solid circles) and presence (empty circles) of protein is shown for $(a)$ apo WT $C k$ ZnuA and (b) $\triangle$ Loop $C k$ ZnuA. Titrations were performed in triplicate and error bars represent the standard deviation between experiments. Fits are shown as solid lines. Dissociation constants \pm standard deviations $(n=3)$ are indicated. 
chains zinc is bound between the domains and is coordinated by Glu59, His60, His147 and His211 (Fig. 2d). This is the same coordination environment as is observed in the Ec ZnuA (Yatsunyk et al., 2008; Li \& Jogl, 2007) and Synechocystis ZnuA (Banerjee et al., 2003) structures, but differs from that observed for Se ZnuA, in which one of the flexible-loop His residues (His140) replaces His60 (Ilari et al., 2011). An anomalous difference map confirms the position of zinc in this structure (Fig. 2e), which is bound in a tetrahedral geometry (Fig. 2f).

\subsection{Differences and similarities between ZnuA proteins}

Like many ZnuA homologues, $C k$ ZnuA is capable of binding multiple zinc ions, which requires the presence of the His-rich loop (Yatsunyk et al., 2008; Ilari et al., 2011, 2014;

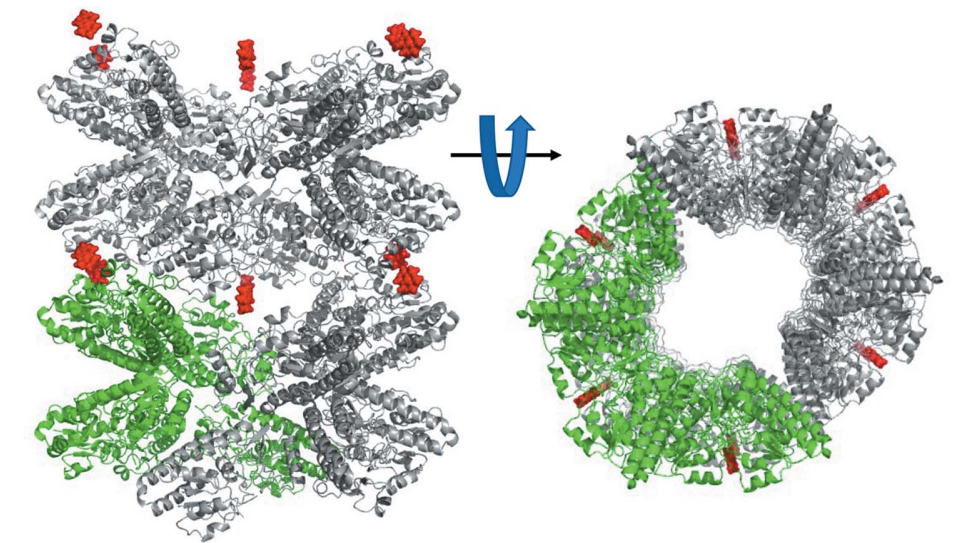

(a)

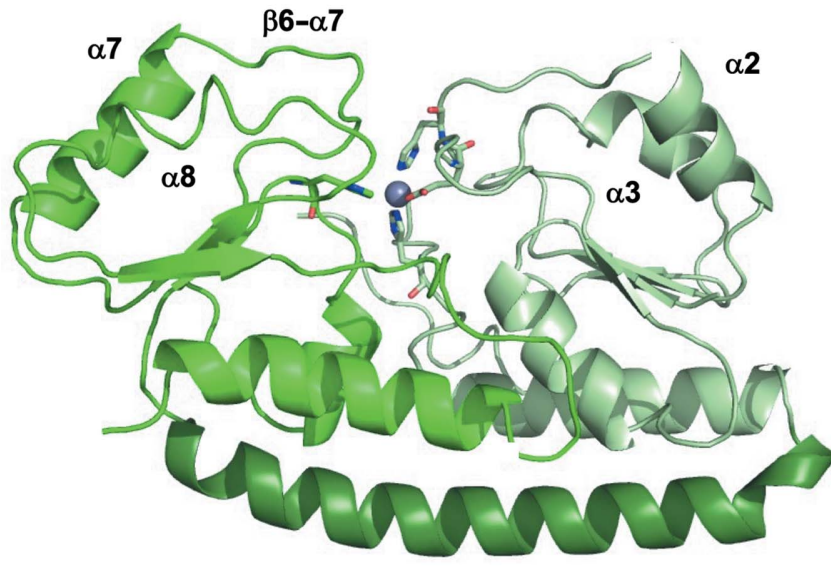

(c)

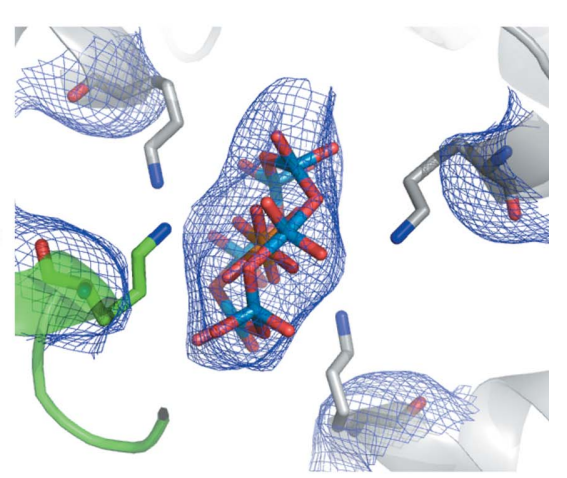

(b)

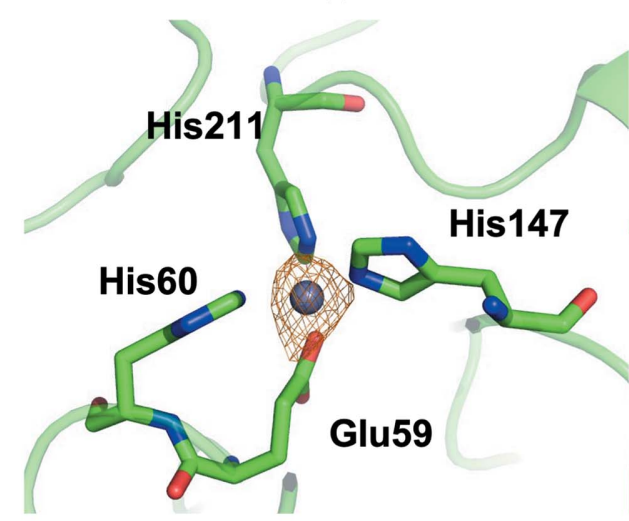

(e)

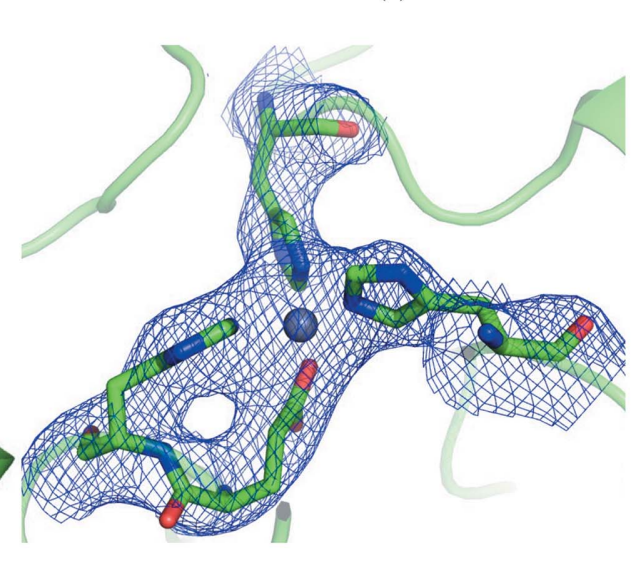

$(d)$

Figure 2

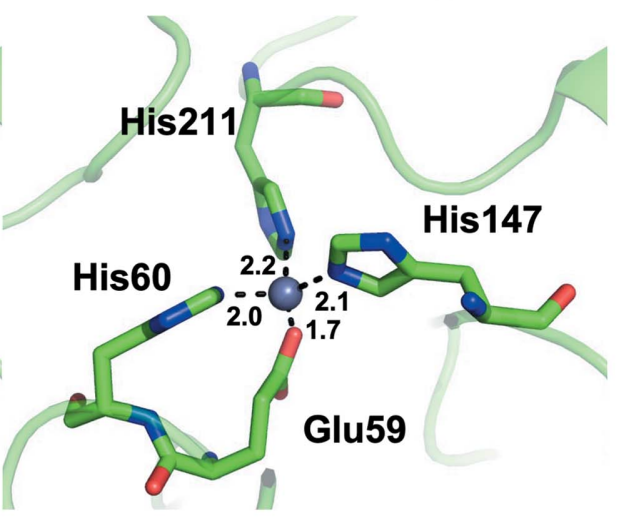

$(f)$

The crystal structure of holo $C k$ ZnuA. (a) The lattice of the $C k$ ZnuA crystals. The protein is shown in ribbon form with one asymmetric unit shown in green and the remaining three in gray. TEW is shown as red spheres. (b) TEW binding site showing TEW as sticks colored according to element (oxygen, red; tungsten, blue; tellurium, orange) and Lys 91 from each of four symmetry-related protein molecules as sticks. $2 F_{\mathrm{o}}-F_{\mathrm{c}}$ electron density is shown as a blue mesh contoured at 1.0.$(c)$ Whole protein shown as a ribbon diagram with the NTD in light green, the linker helix in dark green and the CTD in medium green, with zinc shown as a small gray sphere and ligands shown as sticks colored by element. $(d)$ The zinc-binding site showing $2 F_{\mathrm{o}}-F_{\mathrm{c}}$ electron density contoured at $1.5 \sigma,(e)$ an anomalous difference map contoured at $3.0 \sigma$ and $(f)$ zinc-ligand distances in $\AA$. 


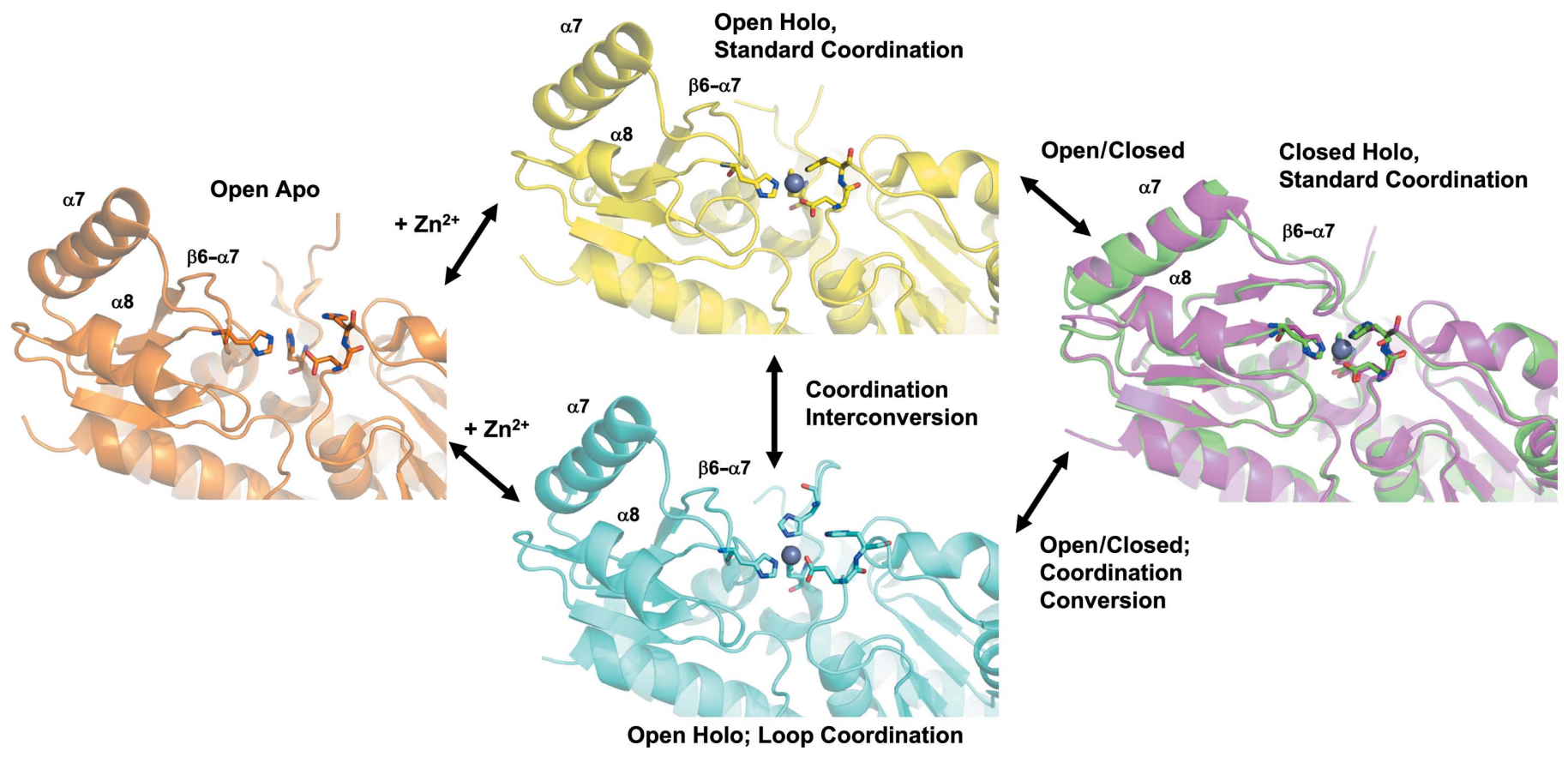

Figure 3

Structural landscape of ZnuA proteins. Representative structures of ZnuA in the open apo form (Ec ZnuA; Hesse et al., 2019; orange; PDB entry 2ps3), the open holo form with coordination by His60 (Ec ZnuA; Hesse et al., 2019; 'Standard Coordination'; yellow; PDB entry 2prs chain B), the open holo form with coordination by loop residue His140 (Se ZnuA; Li \& Jogl, 2007; 'Loop Coordination'; cyan; PDB entry 2xqv chain $B$ ) and the closed holo form ( Ck ZnuA; green; PDB entry 7rcj) aligned with Ec ZnuA (Hesse et al., 2019; magenta, PDB entry 2prs chain $A$ ).

Pederick et al., 2015; Desrosiers et al., 2007; Lu et al., 1997). Although conserved in many zinc-specific cluster A-I SBPs, the function of this loop remains enigmatic. A function as a zinc chaperone to the high-affinity site has been proposed (Banerjee et al., 2003; Desrosiers et al., 2007; Lu et al., 1997), as has a regulatory function as a sensor of high zinc concentrations (Wei et al., 2007). However, little conclusive evidence has been provided for either case. Most of the sequence differences between the ZnuA proteins from C. koseri ( $C k \mathrm{ZnuA})$, E. coli (Ec ZnuA) and S. enterica (Se ZnuA) are found in this region. The $C k$ ZnuA loop allows the binding of two additional zinc ions, while only one zinc-binding event could be ascribed to the loop in Ec ZnuA (Yatsunyk et al., 2008) and Se ZnuA (Ilari et al., 2014). This may be a simple consequence of the presence of two extra His residues in the $C k$ ZnuA loop relative to the other ZnuA homologues discussed here.

What is most remarkable about the structure of $C k \mathrm{ZnuA}$ are the structural differences between it and Se ZnuA, despite the nearly $100 \%$ sequence identity between the proteins. Apart from changes in the loop region, which is disordered in both structures, there are only five very conservative aminoacid substitutions differentiating these proteins. Nevertheless, they adopt different zinc-coordination environments and the conformation of $\mathrm{Se} \mathrm{ZnuA}$ is open while that of $C k \mathrm{ZnuA}$ is closed. $C k$ ZnuA more closely resembles the closed conformation of holo Ec ZnuA, despite their lower sequence identity. Taken together, the structures of ZnuA from these three species suggest a diverse structural landscape and a potential pathway for zinc binding, as outlined below and in Fig. 3 .

The only WT apo structure is that from Ec ZnuA and it exhibits an open conformation. Two zinc-bound structures exist in open forms with different coordination. The zinc in Se ZnuA is coordinated by His 140 of the His-rich loop (Fig. 3, 'Loop Coordination') rather than the absolutely conserved His60 observed to coordinate in Ec ZnuA (Fig. 3, 'Standard Coordination'). $C k$ ZnuA was solved in the holo form in a closed conformation very similar to that of the closed holo Ec ZnuA structure. These structures suggest a zinc-binding pathway whereby ZnuA acquires zinc in the open form first through a loop residue (His140 in Se ZnuA). His60 could then displace this residue, again in the open conformation, followed by closure of the $\beta 6-\alpha 7$ loop and $\alpha 7$ and $\alpha 8$ helices to yield the closed, holo form. However, it is important to note that the loop is not required for high-affinity zinc binding in cluster A-I SBPs, as demonstrated here for $C k$ ZnuA and previously for several others (Wei et al., 2007; Neupane et al., 2017, 2019). Thus, Fig. 3 indicates simply the possibility of the interconversion of metal-coordination states rather than an obligate, directional process. Nevertheless, this conformational diversity among a closely related group of ZnuA proteins reveals a dynamic landscape that is likely to be functionally important and provides several potential targets for rational drug design.

\section{Acknowledgements}

We acknowledge the staff at the Berkeley Center for Structural Biology at Lawrence Berkeley National Laboratory. The Berkeley Center for Structural Biology is supported in part by the National Institutes of Health, National Institute of General Medical Sciences and the Howard Hughes Medical Institute. The Advanced Light Source is supported by the 
Director, Office of Science, Office of Basic Energy Sciences of the US Department of Energy under Contract No. DE-AC0205CH11231. The PILATUS detector on beamline 5.0.1 was funded under NIH grant S10OD021832. The atomic coordinates and structure factors for $C k$ ZnuA (7rcj) have been deposited in the Protein Data Bank, Research Collaboratory for Structural Bioinformatics, Rutgers University, New Brunswick, New Jersey, USA (http://www.rcsb.org/).

\section{Funding information}

Funding for this research was provided by: National Institutes of Health, National Institute of General Medical Sciences (grant No. 1R01GM122819).

\section{References}

Ammendola, S., Pasquali, P., Pistoia, C., Petrucci, P., Petrarca, P., Rotilio, G. \& Battistoni, A. (2007). Infect. Immun. 75, 5867-5876.

Banerjee, S., Wei, B., Bhattacharyya-Pakrasi, M., Pakrasi, H. B. \& Smith, T. J. (2003). J. Mol. Biol. 333, 1061-1069.

Berntsson, R. P., Smits, S. H. J., Schmitt, L., Slotboom, D. \& Poolman, B. (2010). FEBS Lett. 584, 2606-2617.

Bijelic, A., Molitor, C., Mauracher, S. G., Al-Oweini, R., Kortz, U. \& Rompel, A. (2015). ChemBioChem, 16, 233-241.

Bijelic, A. \& Rompel, A. (2017). Acc. Chem. Res. 50, 1441-1448.

Bijelic, A. \& Rompel, A. (2018). ChemTexts, 4, 10.

Boer, M. de, Gouridis, G., Vietrov, R., Begg, S. L., SchuurmanWolters, G. K., Husada, F., Eleftheriadis, N., Poolman, B., McDevitt, C. A. \& Cordes, T. (2019). eLife, 8, e44652.

Davidson, A. L., Dassa, E., Orelle, C. \& Chen, J. (2008). Microbiol. Mol. Biol. Rev. 72, 317-364.

Desrosiers, D. C., Sun, Y. C., Zaidi, A. A., Eggers, C. H., Cox, D. L. \& Radolf, J. D. (2007). Mol. Microbiol. 65, 137-152.

Edelhoch, H. S. (1967). Biochemistry, 6, 1948-1954.

Emsley, P., Lohkamp, B., Scott, W. G. \& Cowtan, K. (2010). Acta Cryst. D66, 486-501.

Golynskiy, M., Gunderson, W. A., Hendrich, M. P. \& Cohen, S. M. (2006). Biochemistry, 45, 15359-15372.

Handali, M., Neupane, D. P., Roychowdhury, H. \& Yukl, E. T. (2015). J. Biol. Chem. 290, 11878-11889.

Handali, M., Roychowdhury, H., Neupane, D. P. \& Yukl, E. T. (2015). J. Biol. Chem. 290, 29984-29992.

Heide, T. van der \& Poolman, B. (2002). EMBO Rep. 3, 938-943.

Hesse, L., Lonergan, Z. R., Beavers, W. N. \& Skaar, E. P. (2019). Infect. Immun. 87, e00746-19.

Higgins, C. F. (1992). Annu. Rev. Cell Biol. 8, 67-113.

Ilari, A., Alaleona, F., Petrarca, P., Battistoni, A. \& Chiancone, E. (2011). J. Mol. Biol. 409, 630-641.

Ilari, A., Alaleona, F., Tria, G., Petrarca, P., Battistoni, A., Zamparelli, C., Verzili, D., Falconi, M. \& Chiancone, E. (2014). Biochim. Biophys. Acta, 1840, 535-544.

Ilari, A., Pescatori, L., Di Santo, R., Battistoni, A., Ammendola, S., Falconi, M., Berlutti, F., Valenti, P. \& Chiancoe, E. (2016). Biochim. Biophys. Acta, 1860, 534-541.

Kabsch, W. (2010a). Acta Cryst. D66, 125-132.

Kabsch, W. (2010b). Acta Cryst. D66, 133-144.

Karplus, P. A. \& Diederichs, K. (2012). Science, 336, 1030-1033.

Karplus, P. A. \& Diederichs, K. (2015). Curr. Opin. Struct. Biol. 34, $60-68$.
Khare, D., Oldham, M. L., Orelle, C., Davidson, A. L. \& Chen, J. (2009). Mol. Cell, 33, 528-536.

Kuzmič, P. (1996). Anal. Biochem. 237, 260-273.

Kuzmič, P. (2006). Mol. Cell. Endocrinol. 248, 172-181.

Kuzmič, P. (2009). Methods Enzymol. 467, 247-280.

Li, H. \& Jogl, G. (2007). J. Mol. Biol. 368, 1358-1366.

Loisel, E., Jacquamet, L., Serre, L., Bauvois, C., Ferrer, J. L., Vernet, T., Di Guilmi, A. M. \& Durmort, C. (2008). J. Mol. Biol. 381, 594606.

Lu, D., Boyd, B. \& Lingwood, C. A. (1997). J. Biol. Chem. 272, $29033-$ 29038.

Luft, J. R., Collins, R. J., Fehrman, N. A., Lauricella, A. M., Veatch, C. K. \& DeTitta, G. T. (2004). J. Struct. Biol. 142, 170-179.

Mac Sweeney, A., Chambovey, A., Wicki, M., Müller, M., Artico, N., Lange, R., Bijelic, A., Breibeck, J. \& Rompel, A. (2018). PLoS One, 13, e0199639.

Mauracher, S. G., Molitor, C., Al-Oweini, R., Kortz, U. \& Rompel, A. (2014). Acta Cryst. F70, 263-266.

McCoy, A. J., Grosse-Kunstleve, R. W., Adams, P. D., Winn, M. D., Storoni, L. C. \& Read, R. J. (2007). J. Appl. Cryst. 40, 658-674.

Murshudov, G. N., Skubák, P., Lebedev, A. A., Pannu, N. S., Steiner, R. A., Nicholls, R. A., Winn, M. D., Long, F. \& Vagin, A. A. (2011). Acta Cryst. D67, 355-367.

Neupane, D. P., Avalos, D., Fullam, S., Roychowdhury, H. \& Yukl, E. T. (2017). J. Biol. Chem. 292, 17496-17505.

Neupane, D. P., Kumar, S. \& Yukl, E. T. (2019). Biochemistry, 58, 126136.

Pederick, V. G., Eijkelkamp, B. A., Begg, S. L., Ween, M. P., McAllister, L. J., Paton, J. C. \& McDevitt, C. A. (2015). Sci. Rep. 5, 13139.

Quiocho, F. A. \& Ledvina, P. S. (1996). Mol. Microbiol. 20, 17-25.

Scheepers, G. H., Lycklama a Nijeholt, J. A. \& Poolman, B. (2016). FEBS Lett. 590, 4393-4401.

Sheng, Y., Fan, F., Jensen, O., Zhong, Z., Kan, B., Wang, H. \& Zhu, J. (2015). Infect. Immun. 83, 3902-3908.

Sobala, Ł. F., Fernandes, P. Z., Hakki, Z., Thompson, A. J., Howe, J. D., Hill, M., Zitzmann, N., Davies, S., Stamataki, Z., Butters, T. D., Alonzi, D. S., Williams, S. J. \& Davies, G. J. (2020). Proc. Natl Acad. Sci. USA, 117, 29595-29601.

Vandebroek, L., Noguchi, H., Kamata, K., Tame, J. R. H., Van Meervelt, L., Parac-Vogt, T. N. \& Voet, A. R. D. (2020). Chem. Commun. 56, 11601-11604.

Wang, Y., Graichen, M. E., Liu, A., Pearson, A. R., Wilmot, C. M. \& Davidson, V. L. (2003). Biochemistry, 42, 7318-7325.

Wei, B., Randich, A. M., Bhattacharyya-Pakrasi, M., Pakrasi, H. B. \& Smith, T. J. (2007). Biochemistry, 46, 8734-8743.

Winn, M. D., Ballard, C. C., Cowtan, K. D., Dodson, E. J., Emsley, P., Evans, P. R., Keegan, R. M., Krissinel, E. B., Leslie, A. G. W., McCoy, A., McNicholas, S. J., Murshudov, G. N., Pannu, N. S., Potterton, E. A., Powell, H. R., Read, R. J., Vagin, A. \& Wilson, K. S. (2011). Acta Cryst. D67, 235-242.

Yang, X., Becker, T., Walters, N. \& Pascual, D. W. (2006). Infect. Immun. 74, 3874-3879.

Yao, Y., Falgenhauer, L., Falgenhauer, J., Hauri, A. M., Heinmüller, P., Domann, E., Chakraborty, T. \& Imirzalioglu, C. (2021). Front. Cell. Infect. Microbiol. 11, 744431.

Yatsunyk, L. A., Easton, J. A., Kim, L. R., Sugarbaker, S. A., Bennett, B., Breece, R. M., Vorontsov, I. I., Tierney, D. L., Crowder, M. W. \& Rosenzweig, A. C. (2008). J. Biol. Inorg. Chem. 13, 271-288. 